control rates (target blood pressure $90 \mathrm{~mm} \mathrm{Hg}$ ) were higher for aliskiren groups (300 mg, 52.46\%; $150 \mathrm{mg}, 49.82 \%$ and $75 \mathrm{mg}$, $45.91 \%$ ) compared to ramipril (5 mg, 44.44\%); and aliskiren $300 \mathrm{mg}$ group was significantly superior to ramipri $15 \mathrm{mg}$ group $(\mathrm{p}=0.0359)$. The overall incidence of adverse effects (AEs) was similar among the treatment groups. The ramipril group had at least a four times higher incidence of cough (6.0\%) compared with the three aliskiren treatments (ranging from $0.4 \%$ to $1.4 \%$ ).

Conclusion Aliskiren was well tolerated, and superior or non-inferior to ramipril in lowering $\mathrm{BP}$ in Chinese patients with essential hypertension.

\section{e0353 PERIPHERAL PULSE WAVE VELOCITY AS SCREENING FOR SUBCLINICAL VASCULOPATHY IN RHEUMATOID ARTHRITIS}

doi:10.1136/hrt.2010.208967.353

${ }^{1} \mathrm{Xu}$ Ting, ${ }^{2}$ Woo Kamsang, ${ }^{3}$ Fang Yongfei, ${ }^{4}$ Huang Jing. ${ }^{1}$ The Second Hospital of Chongqing Medical University; ${ }^{2}$ Department of Biochemistry, The Chinese University of Hong Kong: ${ }^{3}$ Department of Rheumatology, The Southwest Hospital of The Third Military Medical University; ${ }^{4}$ Second Affiliated Hospital of Chongqing Medical University

Objective This study aims to evaluate a noninvasive method for screening of early vasculopathy in high risk patients suffering from rheumatoid arthritis (RA)

Methods Brachial-ankle pulse wave velocity (baPWV) was measured in RA group $(n=132)$ and age, gender, height-matched healthy volunteers $(n=132)$. Vasculitis specific biomarkers, biochemical and immune indices were recorded in RA patients. Multiple regression analysis was performed in RA group to determine the independent predictors of baPWV. Echo-tracking technique was used to measure pressure elastic coefficient (E $\rho)$ and stiffness parameter $(\beta)$ of brachial and posterior tibial artery in 25 RA patients and 48 matched controls.

Results The baPWV in RA group was significantly increased as compared with control group $(15.0[9.2-30.0] \mathrm{m} / \mathrm{s}$ versus 12.6 [9.6-16.0] m/s, respectively, $\mathrm{p}<0.0001)$. On multiple regression, baPWV correlated independently with age, heart rate, mean arterial pressure and perinuclear antineutrophil cytoplasmic antibodies (p-ANCA) $\quad(\mathrm{R} 2 \mathrm{adj}=0.6868, \quad \mathrm{p}<0.0001 ; \quad \mathrm{F}=33.32, \quad \mathrm{p}<0.0001 ;$ power $=85 \% ; n=107)$. The Ep $(477.9 \pm 138.1$ versus $315.2 \pm 111.9$ $\mathrm{KPa} ; \mathrm{p}<0.0001)$ and $\beta(34.90 \pm 11.00$ vs $24.99 \pm 7.81 ; \mathrm{p}<0.0001)$ of posterior tibial artery in RA group were significantly higher than those in control group. There were no significant difference in $\mathrm{E} \rho$ and $\beta$ of brachial artery between the two groups ( $p>0.05)$.

Conclusions There was a significant increase of baPWV, Ep and $\beta$ of posterior tibial artery in RA group compared with control subjects, which related well to p-ANCA. BaPWV may provide a simple, noninvasive method for screening of early subclinical vasculopathy in RA patients.

\section{e0354 ANGIOGRAPHIC CHARACTERISTICS OF PREMATURE CORONARY HEART DISEASE-ANALYSES OF SINGLE CENTER}

doi:10.1136/hrt.2010.208967.354

Gao Lijian, Chen Jun, Chen Jilin, Yang Yuejin, Qiao Shubin, Xu Bo, Li Jianjun, Qin Xuewen, Yuan Jinqing, Wu Yongjian, Chen Jue, You Shijie, Qian Jie, Dai Jun, Hu Fenghuan, Gao Runlin. Fuwai Hospital

Background There are no large-sample published reports prospectively or consecutively assessing the angiographic characteristics of premature coronary heart disease in China.

Objectives The present study was carried out to collect and analyse the clinical and angiographic characteristics in a single center.
Methods From April 2004 to April 2008, based on the screening condition of male $<55$ years and female ( $<65$ years), we selected premature coronary heart disease from a dedicated database of coronary angioplasty registry of the Center for Diagnosis \& Treatment of Coronary Artery Disease, Fuwai Hospital. They had been subjected to coronary angiogram due to the angina pectoris or asymptomatic myocardial ischaemia in coronary heart disease.

Results 4478 consecutive patients (3056 males, 1422 females), average age was $(49.9 \pm 7.0)$ years, were defined as coronary heart disease according to clinical manifestation and coronary angiogram. Patients with unstable angina pectoris (UAP), stable angina pectoris (SAP) and without angina pectoris were 2400, 1534 and 544, respectively. The proportion of coronary heart disease, hypertension, hyperlipidaemia, diabetic mellitus (DM), smoking history, prior myocardial infarction (MI), prior percutaneous intervention (PCI), coronary artery bypass graft and history of cerebrovascular diseases were $6.7 \%, 53.0 \%, 35.3 \%, 20.8 \%, 90.3 \%, 43.3 \%, 16.6 \%, 1.5 \%, 1.1 \%$, respectively. The ratio of eccentric lesions, concentric lesions, chronic total occlusion lesions (CTO) and calcified lesions were $93.7 \%, 23 \%$ and $42.8 \%$, respectively. Comparison between UAP and SAP, there was more MI, PCI, complex lesions $(\mathrm{B} 2+\mathrm{C})$, eccentric lesions and calcium lesions among 3934 patients. There were more MI, PCI, DM, hyperlipidaemia, hypertension, cerebrovascular diseases, smoking, eccentric lesions complex lesions, angulated lesions and calcified lesions in male than that in female (all $\mathrm{p}<0.05$ ). Conclusions The ratio of sex and clinical findings are different in Chinese Premature coronary heart patients. There were more MI, $\mathrm{PCI}$, complex lesions, eccentric lesions and calcified lesions in UAP group, comparison between sexes, there were higher incidences of hypertension, hyperlipidaemia, cerebrovascular disease and smoking history in male.

\section{E0355 CLINICAL OUTCOMES OF PERCUTANEOUS CORONARY INTERVENTION WITH STENT KISSING BALLOON (SKB) TECHNIQUE IN OSTIAL LAD LESIONS}

doi:10.1136/hrt.2010.208967.355

Chen Jilin, Gao Lijian, Chen Jue, Wu Yongjian. Fuwai Hospital

Objective To explore a new technique (stent kissing balloon, SKB) for the treatment of special ostial LAD stenosis.

Methods From January 2008 to March 2010, 8 patients were enrolled to this study, the including entry creteria were left anterior descending artery (LAD) ostial stenosis $>70 \%$, and with the angle between LAD and left circumflex artery (LCX) was less than $60^{\circ}$; left main artery diameter was much larger than LAD ostial diameter $(>1 \mathrm{~mm}$ ), at same time LCX ostial without obvious stenosis lesions. The key point of procedure was as follows: firstly, the stent in LAD and the balloon in LCX should arrive at the positions simultaneously. Secondly, the proximal marker of the balloon was a little ahead of the proximal marker of the stent, then first release the stent with high pressure (12-14 atm) and at last inflate the stent and balloon simultaneously (final kissing with 8-10 atm). Follow-up was carried out by outpatient, phone calls or coronary angiography. Results The patients average age was $54.4 \pm 9.0$ years, 6 was male, 2 was female, 3 patients with diabetes, four patients with hypertension, one patients with prior myocardial infarction, the characteristics of lesions was diffused in two patients and tubular in 6 patients. The average of diameter stenosis was $86 \pm 8 \%$. All the patients accomplished clinical follow-up, average $590 \pm 202$ days (268-810 days), there was no major adverse cardiac events (including target lesion revascularization, myocardial infarction and all-cause death), four patients accomplished 6-8 angiographic follow-up. There was no in-stent restenosis in these four patients. 